\title{
Probing Local lonic Dynamics in Functional Oxides at the Nanoscale
}

\author{
Evgheni Strelcov, ${ }^{* \dagger}{ }^{\dagger}$ Yunseok Kim, ${ }^{\|, \dagger}$ Stephen Jesse, ${ }^{\dagger}$ Ye Cao, ${ }^{\S}$ Ilia N. Ivanov, ${ }^{\dagger}$ Ivan I. Kravchenko, ${ }^{\dagger}$
} Chih-Hung Wang, ${ }^{\ddagger}$ Yung-Chun Teng, ${ }^{\ddagger}$ Long-Qing Chen, ${ }^{\S}$ Ying Hao Chu, ${ }^{\ddagger}$ and Sergei V. Kalinin ${ }^{* \dagger}$

${ }^{\dagger}$ Center for Nanophase Materials Sciences, Oak Ridge National Laboratory, Oak Ridge, Tennessee 37831, United States

${ }^{\ddagger}$ Department of Materials Science and Engineering, National Chiao Tung University, Hsinchu 30010, Taiwan

${ }^{\S}$ Department of Materials Science and Engineering, The Pennsylvania State University, University Park, Pennsylvania, 16802, United States

"School of Advanced Materials Science \& Engineering, Sungkyunkwan University, Suwon, Gyeonggi-do 440-746, Republic of Korea

\section{Supporting Information}

ABSTRACT: A scanning probe microscopy technique for probing local ionic dynamics in electrochemically active materials based on the first-order reversal curve current-voltage (FORC-IV) method is presented. FORC-IV imaging mode is applied to a Ca-substituted bismuth ferrite (Ca-BFO) system to separate the electronic and ionic phenomena in this material and visualize the spatial variability of these behaviors. The variable-temperature measurements further demonstrate the interplay between the thermally and electric-fielddriven resistance changes in Ca-BFO. The FORC-IV is shown to be a simple, powerful, and flexible method for studying electrochemical activity of materials at the nanoscale and, in conjunction with the electrochemical strain microscopy, it can be used for

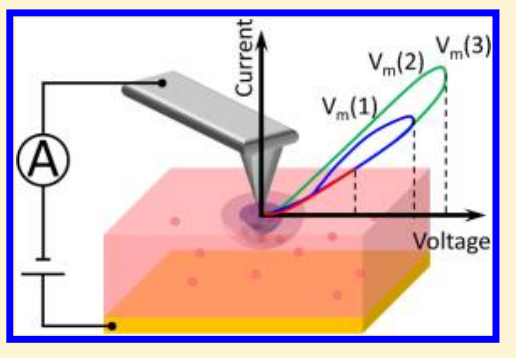
differentiating ferroelectric and ionic behaviors.

KEYWORDS: SPM, ionic dynamics, Ca-BFO, voltage spectroscopy, oxygen vacancy, FORC-IV

A broad gamut of electronic, magnetic, transport, and ferroic properties of transition metal oxides (TMOs) have attracted significant attention to these materials both from the perspective of basic physics ${ }^{1-3}$ and applications. ${ }^{4-6}$ Of particular interest are cross-coupled phenomena including bulk and interface-mediated magnetoelectric couplings, ${ }^{7-10}$ strain controlled metal-insulator and magnetic transitions, ${ }^{11-13}$ and others. Unique properties of TMOs allow for new discoveries of both fundamental and practical importance, for instance, recently shown ${ }^{14,15}$ mechanical control over ionic dynamics and resistive switching that can spur the development of novel data storage technologies.

Notably, many TMOs classes including manganites, ${ }^{16}$ cobaltites, ${ }^{17,18}$ and ferrites, ${ }^{19,20}$ explored in the context of physical behavior are also ionic or mixed electronic-ionic conductors, which underpins applications in energy conversion technologies, for example, solid oxide fuel cells. ${ }^{21,22}$ Given the governing role of the oxidation state of the transition metal cation in the oxide properties, ionic behavior of the TMOs can be an effective way to control the physical properties of materials. $^{23}$ Illustrating this concept, ionically controlled metal-insulator transitions are now rapidly explored in memristive and neuromorphic electronics ${ }^{24-28}$ as well as charge writing. ${ }^{29-31}$ Electrochromic behavior in materials such as doped $\mathrm{WO}_{3}$ and $\mathrm{BiFeO}_{3}, \mathrm{NiO}$ exemplifies the coupling between ionic and optical properties. ${ }^{32,33}$ Similarly, a family of interesting phenomena emerges due to the interplay between ionic and ferroelectric behaviors. ${ }^{33,34}$

The possibility of ionic control over a material's functionality brings forth the challenge of addressing these phenomena locally and with high veracity. ${ }^{23}$ The first requirement stems both from the sub-100 $\mathrm{nm}$ device sizes and length scales of the interactions underpinning ionic functionalities, including characteristic size of defects and corresponding screening and correlation lengths. The second requirement is the need for separation of dissimilar functionalities, for example ionic transport, polarization-driven responses, and surface electrochemistry. ${ }^{35,36}$

Recently, we have developed the electrochemical strain microscopy (ESM) approach for probing ionic dynamics in solids based on voltage-strain coupling, ${ }^{37-41}$ building on the earlier work on piezoresponse force microscopy (PFM). ${ }^{42-46}$ However, ESM/PFM does not allow for differentiating between the ionic and ferroelectric polarization responses, leading to ambiguity in the interpretation of data related to incipient and quantum ferroelectrics, ${ }^{47,48}$ and ultrathin ferroelectric films. Furthermore, the applicability of these techniques can be limited for materials with significant electronic conductivity, where the electrochemical reaction zone can be delocalized and leakage currents can result in additional Joule heating effects. ${ }^{49}$

Here, we describe a newly developed scanning probe microscopy-based (SPM) method for probing local ionic dynamics in mixed electronic-ionic conductors based on the first order reversal curve (FORC) current-voltage (I-V)

Received: December 11, 2012

Revised: July 15, 2013

Published: July 18, 2013 
measurement. This FORC-IV imaging and spectroscopy is applied to a Ca-substituted $\mathrm{BiFeO}_{3}$ thin film.

I. Principles of FORC-IV Measurements. The basic concept of the FORC-IV method is illustrated in Figure $1 .^{50}$

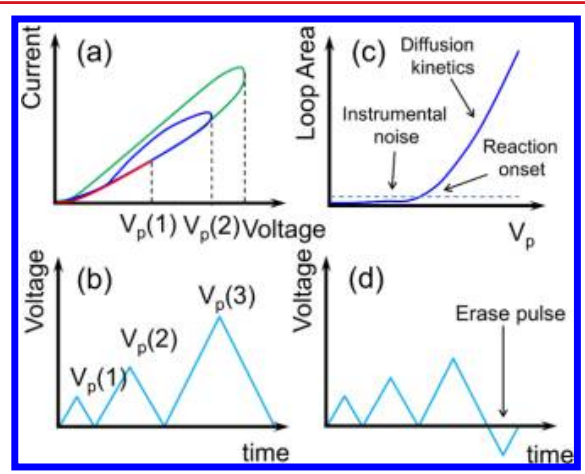

Figure 1. The principle of the FORC-IV method for mapping of local electrochemical reactivity: (a) $I-V$ curves taken as a response to unipolar voltage waveform shown in (b); (c) $I-V$ curves loop areas plotted vs peak biases of each waveform tooth $\left(V_{\mathrm{p}}\right)$; (d) a unipolar voltage waveform can be supplemented with a preset or triggeractivated "erase" pulse at the end.

Here, the current through the tip-surface junction is measured as a function of the tip bias waveform formed by a sequence of triangular pulses with increasing amplitude (peak biases, $V_{\mathrm{p}}$ ). The waveform can be unipolar (shown in Figure $1 \mathrm{~b}$ ), or bipolar. As the tip bias is changed over time according to the waveform, a variety of electronic, ionic, and electrochemical processes can be initiated in the sample. Depending on the rate and reversibility of the processes, this will give rise to nonhysteretic (fast reversible processes) or hysteretic (slow or irreversible) $I-V$ curves. If the RC (Resistance-Capacitance) time constant of the measurement circuit is well below the measurement bandwidth, hysteresis may appear due to ionic dynamics, electrochemical process in the sample, or to Joule heating at the tip-surface junction. We note that hysteresis due to Joule heating can be ascertained and accounted for based on estimates of dissipated power and knowledge of thermal conductivities of the sample and tip ${ }^{49,51}$ and is expected to be minor. Therefore, below we consider bias-induced ionic and electrochemical effects as the predominant source of hysteresis.

For low voltages, the application of bias to the tip results in electronic current flow though the tip-surface junction, but it can be expected not to alter the chemical state of the system at all, or limit the electrochemical changes to fully reversible and rapid (e.g., ionic polarization at the tip-surface junction). In this case, the $I-V$ curve is nonhysteretic (Figure la red curve). For higher biases, the current can induce changes in the chemical or physical state of the material, including transport of mobile ionic species, surface electrochemical reactions, or amorphization-crystallization. These processes are generally slow and are associated with significant kinetic or thermodynamic hysteresis, which, in turn, can be linked to the change in conductivity. Correspondingly, the $I-V$ curve becomes hysteretic in nature (Figure la blue and green curves). Hence, plotting the loop opening as a function of the peak bias will allow establishment of the onset and kinetics of the process (Figure 1c).

The FORC-IV curves can be obtained across a spatially resolved grid of points, giving rise to spectroscopic imaging. The spatial resolution of this FORC-IV SPM is determined by the localization of reaction zone, kinetics, and reversibility of the local electrochemistry. For irreversible processes, the spectroscopic measurements are possible only on sparse grids for which the spacing between measurement points exceeds that of the lateral size of the area modified during the experiment. For these, the use of an active feedback systems (data acquisition is stopped once hysteresis loop opens) can, in principle, allow minimization of the affected area, and hence, increase the spatial resolution. For fully reversible and moderately reversible systems (for which damage is often much more localized than the area that undergoes the reversible transformations), spectroscopic imaging is possible on a dense grid, in which the pixel spacing can be significantly smaller than the size of the signal generation volume, allowing for high-resolution imaging studies. For reversible measurements taken on a dense grid, an "erase" pulse at the end of the waveform can help restore the initial state of the pixel and its surroundings, eliminating the memory of adjacent pixels (e.g., Figure 1d). Such "erase" pulses can be either preset or feedback-activated.

II. FORC-IV Mapping of Reversible Electrochemical Processes. Implementation of the FORC-IV method, was done using $\mathrm{Ca}$-substituted $\mathrm{BiFeO}_{3}$. This material exhibits a fascinating interplay between ferroelectricity and ionic effects and can serve as a model system for studies of coupling between the two. The parent oxide, bismuth ferrite, is a wellknown multiferroic with a ferroelectric Curie temperature $\left(T_{\mathrm{C}}\right)$ of $830{ }^{\circ} \mathrm{C}$ and Neel temperature of $367^{\circ} \mathrm{C} . .^{52}$ Its epitaxial thin films exhibit substrate-induced strain, which stabilizes monoclinic crystalline structure at room temperature. A structural transition to pseudotetragonal phase takes place at around $T_{C}$. Substitution of bismuth with divalent alkaline-earth ions decreases the transition temperature until a local minimum associated with a ferroelectric instability is reached, which lies at $\sim 10 \%$ of substitution ratio for the case of calcium. ${ }^{52}$ Because of high stability of iron oxidation state $(+3)$, Ca-substitution leads to the formation of vacancies, whose concentration is proportional to the substitution level and is responsible for $\mathrm{p}$ type conductivity in Ca-BFO. The instability region is known for a prominent resistive switching behavior with a conductivity change of up to 3 orders of magnitude observed at elevated temperatures. The switching mechanism is still unclear and might be related to bias-induced oxygen vacancy dynamics, electrochemical reactions on the air-solid interface or a combination of the two. As has been shown recently by in situ TEM studies, ${ }^{33}$ application of bias to $10 \%$ Ca-BFO leads to melting of the oxygen vacancy ordering with probable formation of vacancy-rich and vacancy-poor regions that possess different types of main carriers. The moderately slow reversible resistive switching behavior made this material a very attractive test sample for the proof-of-concept of FORC-IV spectroscopic mode performed on a moderately dense grid.

Here, the test sample was a $100 \mathrm{~nm}$ film of $10 \% \mathrm{Ca}$ substituted bismuth ferrite (Ca-BFO) grown on $\mathrm{SrRuO}_{3}$ buffered $\mathrm{SrTiO}_{3}$ substrate with strontium ruthenate acting as a bottom electrode. ${ }^{52}$ The used substitution level stabilizes the pseudotetragonal crystallographic phase of Ca-BFO, which can exist in two conductive states, with about $2-3$ orders of magnitude difference in conductivity between the two. Biasinduced switching between the states is a gradual and reversible process presumably governed by redistribution of oxygen vacancies within the oxide. Thus, although the limiting conductivities are well-defined, there exist a whole range of 
states of intermediate conductivity, some of which are presumably stabilized naturally in the film defects during the growth process. Application of the FORC-IV SPM to this material on the pristine and resistively switched surfaces will, thus, both allow establishing it as a tool for probing the local electronic, ionic and electrochemical activity, as well as provide insight into the electrochemical phenomena on the $\mathrm{Ca}-\mathrm{BFO}$ surfaces.

The current-voltage measurements have been performed on Cypher, MFP-3D (Asylum Research) and Multimode (Bruker) atomic force microscopes (AFMs) interfaced with National Instrument cards controlled by LabView/Matlab software. Cantilevers with conductive boron-doped diamond-coated tips (NT-MDT) were used. In order to eliminate the possibility of irreversible surface electroforming associated with large-scale topographic changes at current $>\sim 100 \mathrm{nA}$, a $200 \mathrm{M} \Omega$ protective resistor was connected in series with the sample.

Figure 2a presents a topographic map of an elliptically shaped surface defect on the Ca-BFO film. The corresponding

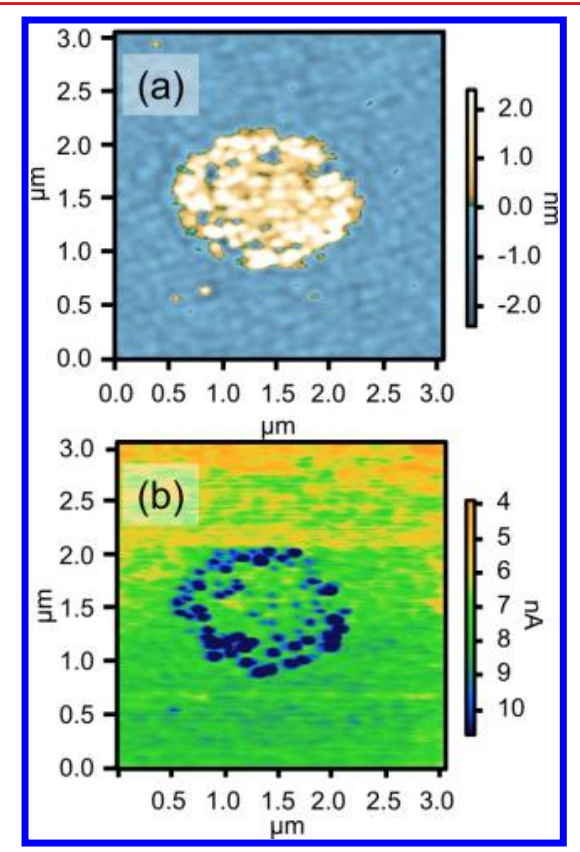

Figure 2. Ca-BFO system: (a) topography of a surface defect, (b) current map of the defect (taken at $-4 \mathrm{~V}) . T=100{ }^{\circ} \mathrm{C}$.

current map (Figure 2b) reveals an annular distribution of the high-conductive state grains. The chosen $3 \times 3 \mu \mathrm{m}^{2}$ region was scanned with a negatively biased conductive tip and the current response of the film to the bias waveform $(1-13 \mathrm{~V}, 11$ peaks, Figure 3a inset) was measured at all locations of a $60 \times 60$ grid. Response averaged over whole grid is presented in the Figure 3a. As expected, $I-V$ loop opening began past a certain threshold voltage $(-2.2 \mathrm{~V}$ in this case, see Figure $3 \mathrm{~b})$. In order to render loop areas taken at different teeth of the waveform comparable, they were normalized to the product of the peak bias and peak current. Below, we refer to this normalized response as the "relative loop area".

The behavior of the average loop area and relative average loop area are very different; whereas the former increases approaching some saturation value as peak bias increases, the later first reaches a maximum and then drops down to almost zero (Figure $3 \mathrm{~b}$ ). We observed that prolonged unipolar bias sweeping at one location leads to switching to the high-

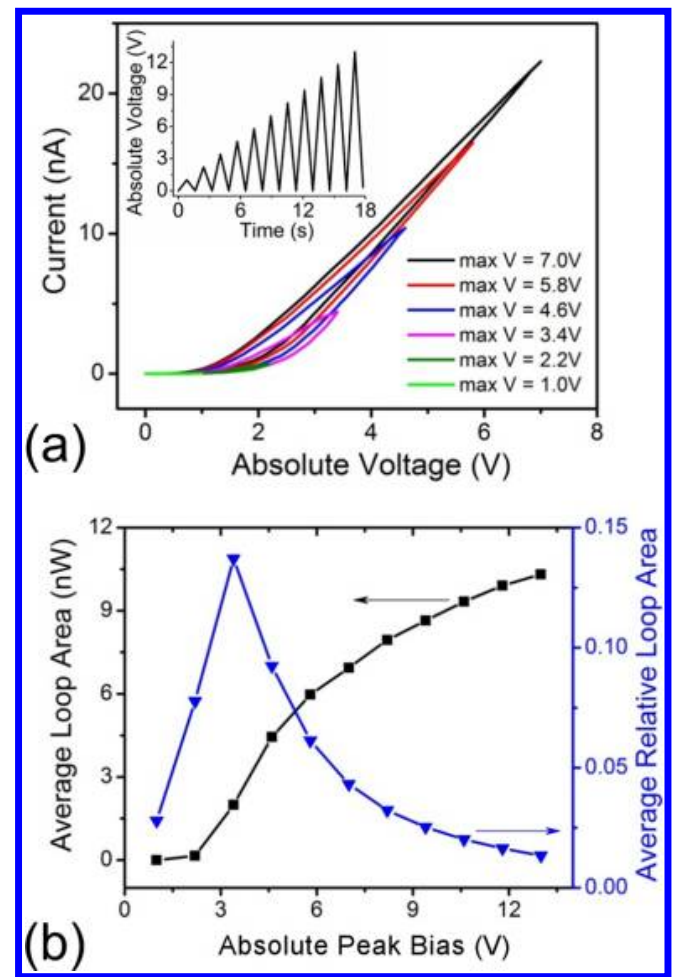

Figure 3. Example of the unipolar spectroscopic mode as applied to the Ca-BFO system: (a) first six average current loops measured in response to the bias waveform of the inset on the film area shown in Figure 2, (b) average absolute and relative loop area as functions of the peak bias. $T=100^{\circ} \mathrm{C}$.

conductive state, and next to no loop opening ensues. This fact can be explained by the predominantly electronic conductivity of the high-conductive state, as opposed to ion-controlled resistivity changes in the low-conductive state. Hence, it follows that the spectroscopic maps acquired at different peak biases cannot be equivalent - there exists an optimal peak bias value at which the measurement-induced disturbance of the sample is minimal and sensitivity of the technique to the electrochemical activity difference between locations is maximal. This indeed was observed; at low and high sweeping biases spectroscopic maps look almost uniform, with no distinguishable features, whereas at intermediate voltages (around $3 \mathrm{~V}$ ) images of highcontrast are produced (Figure 4). This contrast can be directly attributed to the difference in electrochemical behavior at different locations. The spectroscopic maps shown in Figure 4 plot the relative area loop for each individual point of the $60 \times$ 60 grid. Note that although in this case spectroscopic maps look similar to the current AFM maps (due to the inherent linkage between the oxygen vacancy dynamics and conductivity in Ca-BFO), they reveal very different type of information. Specifically, electrochemical activity can be directly visualized in the loop area maps, but cannot be directly extracted from the current map.

To emphasize this point Figure $5 \mathrm{a}, \mathrm{b}$ demonstrates $I-V$ curves taken at two different locations with similar final conductivities yet very different hysteresis behavior. As can be seen, during bias application initial low conductive state switches to higher (Figure $5 b$, lower $I-V$ branch) with the reversal curve deviating significantly from the forward curve and giving rise to loop opening. On the contrary, location that manifested a high conductive state from the very beginning 


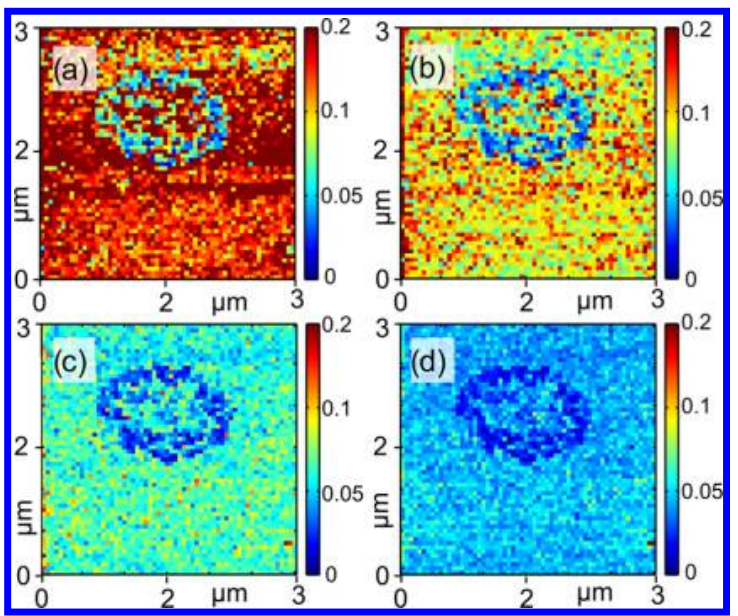

Figure 4. Spectroscopic maps of a Ca-BFO film defect taken at different peak biases: (a) $-3.4 \mathrm{~V}$, (b) $-4.6 \mathrm{~V}$, (c) $-5.8 \mathrm{~V}$, (d) $-7 \mathrm{~V}$; maps plot relative loop area at each pixel of the $60 \times 60$ grid that corresponds to a $3 \times 3 \mu \mathrm{m}^{2}$ surface region; equal colormap scales are used for convenience of comparison; note that maximal contrast is achieved at $-3.4 \mathrm{~V} . \mathrm{T}=100{ }^{\circ} \mathrm{C}$.
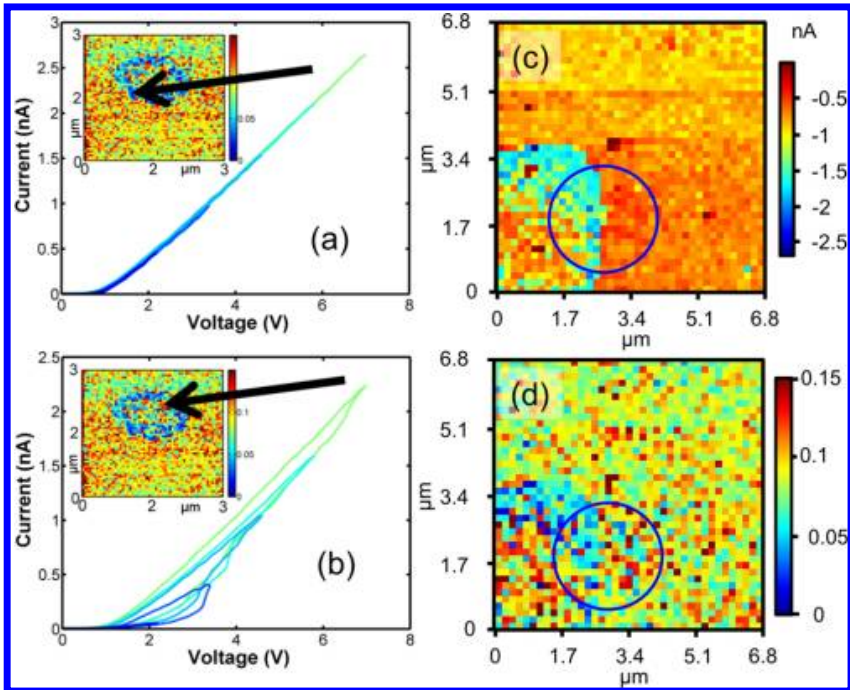

Figure 5. Difference in information contained in CAFM and FORCIV loop area maps. $I-V$ curves taken at different locations indicated with arrows in relative loop area maps of the insets $\left(T=100^{\circ} \mathrm{C}\right)$ : $(\mathrm{a})$ a pixel with initial high conductive state manifests no hysteresis, (b) a pixel with initial low conductive state shows loop opening. Another region with a defect $\left(\mathrm{T}=23^{\circ} \mathrm{C}\right)$ : (c) current map taken at $-6 \mathrm{~V}$ and (d) relative loop area map taken at $-6 \mathrm{~V}$ peak bias showing difference in encircled area.

retains high conductivity levels and displays weak if any hysteresis (Figure 5a). Thus, the loop opening spectroscopic map presents information on the local oxygen vacancy dynamics responsible for the resistive switching, whereas current AFM image of Figure $2 \mathrm{~b}$ only shows distribution of total conductivity in the region of interest at a specific bias. The difference between current map and loop area map is also clearly visible in Figure 5c,d, presenting data for another surface defect. The current map at peak bias $-6 \mathrm{~V}$ (Figure 5c) has a sharp boundary between regions of different conductivity (blue circle). On the contrary, the FORC-IV loop area map (Figure 5d) has a diffuse boundary with interwoven domains (encircled). Supporting Information Figure S1 presents analysis of the current-loop are map difference at the level of individual pixels. It should be noted, that FORC-IV spectroscopic measurements performed on a platinum film sample with a protective resistor of $200 \mathrm{M} \Omega$ yielded ohmic $I-V$ s with no hysteresis. The RC-constant of this system was $8-10 \mathrm{~ms}$ compared to $40 \mathrm{~ms}$ of the voltage step duration.

To establish the role of irreversible and kinetically limited effects on FORC-IV data, we perform imaging of the macroscopic resistively switched areas. These studies further allow excluding potential artifacts associated with the surface topography and demonstrate FORC-IV spectroscopy over large areas. Here, we apply FORC-IV SPM to a predominantly flat region of the sample with directly written distribution of conductive states. A $5 \times 5 \mu \mathrm{m}^{2}$ region of Ca-BFO film was poled at $-15 \mathrm{~V}$ twice to switch it to high conductive state. A square $2.5 \times 2.5 \mu \mathrm{m}^{2}$ centered at that region was then twice poled at $+5 \mathrm{~V}$, reversing the process. Figure $6 \mathrm{a}$ presents a

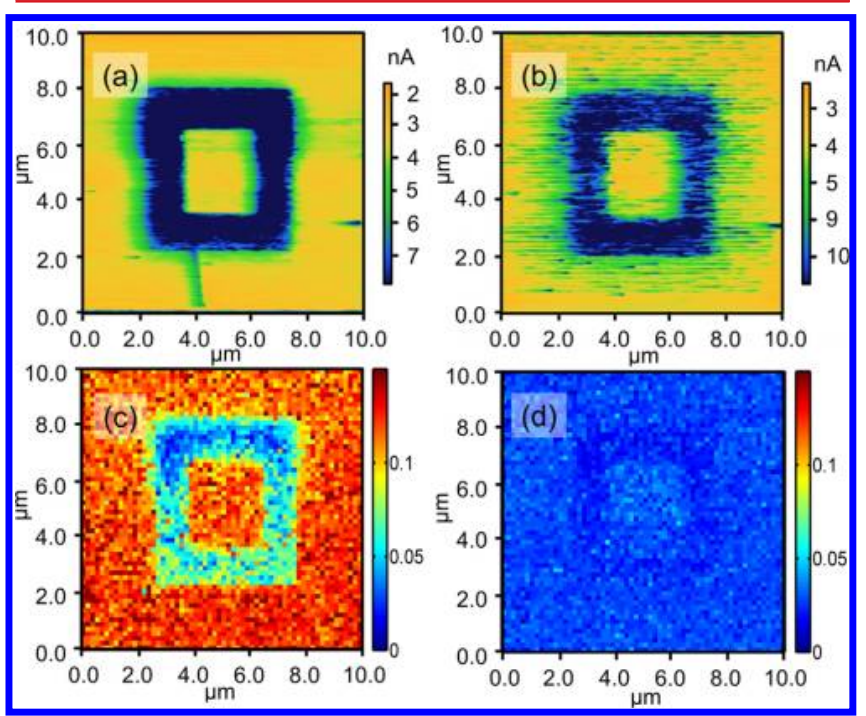

Figure 6. Directly written conductive box pattern on Ca-BFO: (a) CAFM image taken at $-4 \mathrm{~V}$, the bigger box was poled at $-15 \mathrm{~V}$, the inner box - at $+5 \mathrm{~V}$; (b) C-AFM image of same region taken after FORC-IV measurements at $-4 \mathrm{~V}$; (c) spectroscopic relative loop area map at $-3.4 \mathrm{~V}$ peak bias; (d) same as (d) at $-9.4 \mathrm{~V}$ displays almost no contrast; $60 \times 60$ grid of the spectroscopic maps correspond to $10 \times$ $10 \mu \mathrm{m}^{2}$ region of the surface. $\mathrm{T}=100{ }^{\circ} \mathrm{C}$.

current AFM map of the obtained pattern taken at $-4 \mathrm{~V}$ and showing almost complete reversibility of the resistive switching, which allowed us to use moderately dense grid of $60 \times 60$ for unipolar FORC-IV spectroscopy. Another current AFM map, shown in Figure 6b, was acquired after the FORC-IV measurements and clearly shows an increase in conductivity at locations to which unipolar bias waveform had been applied (green stripes in Figure 6b). As explained above, this should lead to worsening of the electrochemistry-related contrast in spectroscopic maps taken at high peak biases (Figure 6c,d). Whereas at optimal peak bias $(-3.4 \mathrm{~V}$ Figure 6c), the spectroscopic map clearly shows significant difference between regions of high conductivity but low electrochemical activity and regions of low conductivity but strong ionic dynamic, the map taken at $-9.4 \mathrm{~V}$ (Figure 6d) is almost featureless.

Hence, the FORC-IV method allows the exploration of the evolution of the extant and predefined ionicaly controlled conductive states in the material. We further note that in contrast to ESM/PFM signals that originate from piezoelectric/ 
Vegard expansion, FORC-IV loops hysteresis is only due to the changes in resistivity that are inherently linked (in the materials in question) to the ionic motion. Thus, FORC-IV truly probes local ionic dynamics.

III. Variable-Temperature Studies. The FORC-IV method allows investigation of the interplay between the thermally- and electrically activated processes (such as diffusion and polarization) that govern resistive switching or any other electrochemical activity in the sample under test. Here, we performed variable-temperature measurements on $\mathrm{Ca}-\mathrm{BFO}$ sample in the range of temperatures $23-162{ }^{\circ} \mathrm{C}$ with the same unipolar waveform applied to the points of a $5 \times 5$ grid on $5 \times 5 \mu \mathrm{m}^{2}$ regions at each temperature. Figure 7 a presents

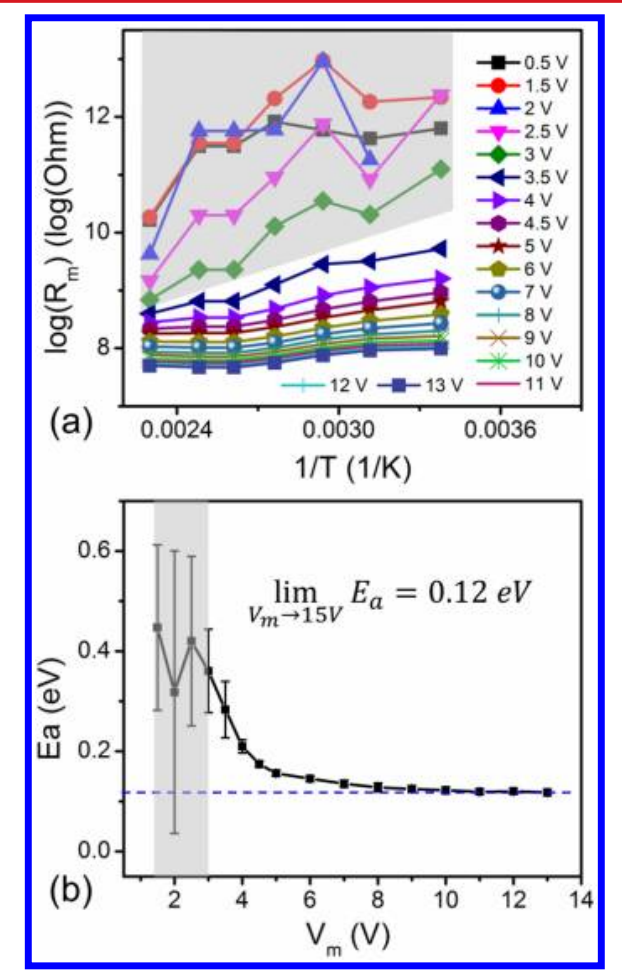

Figure 7. Variable temperature FORC-IV for Ca-BFO sample: hole transport (a) Arrhenius plots of the minimal film resistance for different peak biases, (b) activation energies of the switching process calculated from the Arrhenius plots as a function of peak bias. Dashed line is for eye guidance. Gray shaded areas highlight data with low signal-to-noise ratio. an Arrhenius plot of the average minimal resistance of film $\left(R_{\mathrm{m}}\right)$ measured at each of the peak biases of the voltage waveform. This value was calculated by dividing the corresponding peak bias by the maximal recorded current with further correction for the protective resistor. At peak biases lower than $-2 \mathrm{~V}$, changes in $R_{\mathrm{m}}$ are noise-dominated, as measured currents were below $1 \mathrm{nA}$. Above $-2 \mathrm{~V}$ the $R_{\mathrm{m}}$ dependence on temperature progressively approaches a certain form that manifests two extreme regimes, which have very weak temperature dependence and a switching regime between the two with a distinct activation energy. The latter (Figure $7 \mathrm{~b}$ ) decreases with increasing maximum loop bias, approaching a value of $0.12 \mathrm{eV}$, which is much lower than a typical potential barrier associated with migration of oxygen vacancies in oxides or energy of oxygen vacancy formation (both $\sim 1 \mathrm{eV}$ ) as measured at temperatures larger than $300{ }^{\circ} \mathrm{C} .{ }^{53,54}$ Hence, we pose that this activation energy is associated with the transport of holes in Ca-BFO, rather than motion of oxygen vacancies. Indeed, since $R_{\mathrm{m}}$ is the minimal resistance of the film at a given peak bias, in the limit, when the film has switched to high conductive state, $R_{\mathrm{m}}$ temperature dependence reflects the thermal activation of the hole motion, not that of oxygen vacancies.

To further investigate the thermal activation of the hole transport in Ca-BFO and corroborate the FORC-IV data, macroscopic transport experiments have been performed on a Ca-BFO film grown on $\mathrm{SrTiO}_{3}$ without a bottom electrode. To perform transport measurements, a set of lateral $\mathrm{Cr} / \mathrm{Au}$ electrodes with $100 \mu \mathrm{m}$ interelectrode distance was deposited on top of a $100 \mathrm{~nm}$ film. Impedance spectroscopy was used to determine resistance of the insulating Ca-BFO phase. Bias of 1 $\mathrm{V}$ alternating current (ac) was applied between a pair of electrodes, as frequency was swept from $10 \mathrm{MHz}$ to $0.01 \mathrm{~Hz}$. For the small probing bias, the film conductivity does not change even in the direct current $(\mathrm{dc})$ limit (electric field of the same strength in case of FORC-IV measurements would be created by $1 \mathrm{mV})$. Thus, the temperature dependence of impedance (Figure 8a) reflects hole transport in the insulating Ca-BFO. The shape of the Nyquist plots at different temperatures does not show any diffusional component, but rather can be modeled with a single parallel RC element. The values of resistance extracted from these are equivalent (but, of course, not equal in magnitude) to $R_{\mathrm{m}}$ values of Figure $7 \mathrm{a}$ measured at low biases. To go to the other extreme and

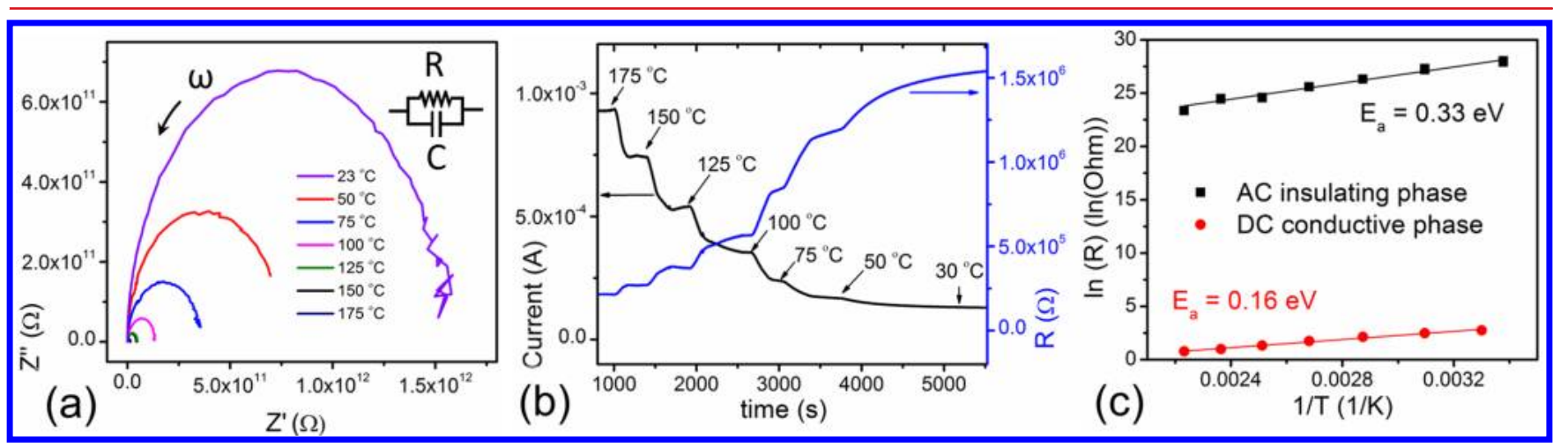

Figure 8. Variable temperature measurements for Ca-BFO sample with lateral electrodes: hole transport (a) Nyquist plot reactance vs resistance of insulating phase showing fitting model in the inset and the frequency increase direction with the arrow, (b) current and resistance vs time curve of the conductive phase taken at a dc bias of $200 \mathrm{~V}$ and with a protective resistor of $100 \mathrm{k} \Omega$, (c) Arrhenius plots of the resistances of insulating (measured with ac) and conductive (measured with dc, protective resistance subtracted) phases. 
determine resistance of the conductive phase, the sample was heated to $175^{\circ} \mathrm{C}$ and $200 \mathrm{~V}$ of dc bias was applied across lateral electrodes for about $8 \mathrm{~h}$ until rising current leveled off at a maximal value. A $100 \mathrm{k} \Omega$, protective resistor was included in the circuit to prevent film's electrical failure. The temperature, then, was lowered in steps, while keeping the bias constant. In this way, applied electric field prevented oxygen vacancies from redistribution and kept the film in the high-conductive state. The total current and resistance curves are shown in Figure $8 \mathrm{~b}$.

The Arrhenius plot of Figure $8 \mathrm{c}$ summarizes temperature data on the insulating and conductive phases and shows activation energies of 0.33 and $0.16 \mathrm{eV}$ for the two of them, respectively. These values are close to the activation energies obtained by the FORC-IV measurements (Figure 7b). Note, however, that the FORC-IV technique allowed probing the activation processes of not only two extremal phases but also of the intermediate states between the two.

Further information on the oxygen vacancy motion or other electrochemical processes could be extracted from the temperature dependence of the FORC-IV loop areas. The average loop area vs peak bias curves shown in Figure 9a, can be fitted

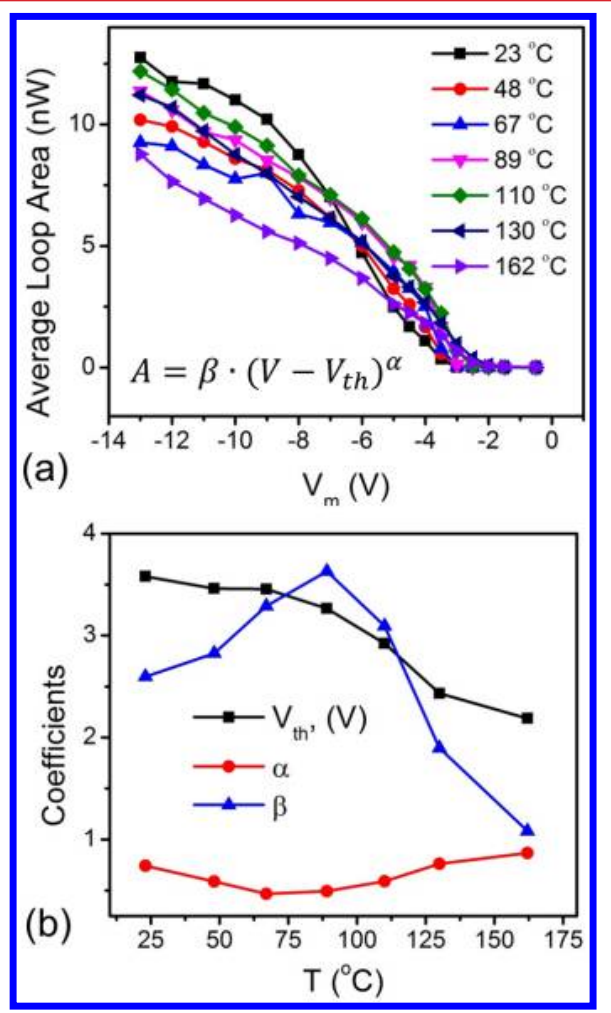

Figure 9. Variable temperature FORC-IV for Ca-BFO system: electrochemistry (a) average $I-V$ loop area vs peak bias for different temperatures, (b) fitting parameters of the function shown in (b) plotted vs temperature; threshold voltage $V_{\text {th }}$ exponent $\alpha$, and coefficient $\beta$.

with a function $\mathrm{A}=\beta\left(V-V_{\mathrm{th}}\right)^{\alpha}$, where $A$ is the average loop area, $V_{\text {th }}$ is the threshold voltage, $\beta$ is a constant, and $\alpha$ is a critical exponent. The choice of the fitting function, generally used for description of critical phenomena, can be justified by noting that the resistive switching in $\mathrm{Ca}-\mathrm{BFO}$ is linked to the order-disorder transition of the oxygen vacancy melting. ${ }^{33}$ Figure $9 \mathrm{~b}$ presents dependence of the fitting parameters on temperature. As expected, the threshold voltage decreases with increasing temperature due to increase in the ion mobility. On the other hand, the power and the proportionality coefficients manifest a nontrivial behavior having extrema around $90{ }^{\circ} \mathrm{C}$.

While the detailed analysis of the exact form of $I-V$ curves and especially FORC loop opening data is hampered by the lack of appropriate theoretical models, some insight into the underpinning mechanisms can be obtained from the threshold voltage corresponding to the onset of electrochemical processes. Here, we consider the redistribution of oxygen vacancies (electrode polarization) maintaining total number of vacancies as one such process. An alternative is the voltageactivated oxygen reduction reaction at the tip-surface junction associated with the change of the total number of vacancies:

$$
\begin{aligned}
& \frac{1}{2} \mathrm{O}_{2}+V_{\mathrm{O}}^{*}+2 \mathrm{e}^{\prime} \rightarrow 2 \mathrm{O}_{\mathrm{O}}^{\times} \\
& \quad \text { (tip-surface junction, cathode) } \\
& \mathrm{Fe}_{\mathrm{Fe}}^{\times}-\mathrm{e}^{\prime} \rightarrow \mathrm{Fe}_{\mathrm{Fe}}^{\cdot} \quad \text { (bottom electrode, anode) } \\
& \mathrm{Ca}_{x} \mathrm{Bi}_{1-x} \mathrm{FeO}_{3-x / 2}(\mathrm{~s})+x \mathrm{O}_{2}(\mathrm{~g}) \rightarrow \mathrm{Ca}_{x} \mathrm{Bi}_{1-x} \mathrm{FeO}_{3}(\mathrm{~s}) ;
\end{aligned}
$$$$
\Delta G_{\mathrm{r}} \text { (overall process) }
$$

The temperature dependence of the Gibbs free energy is $\Delta G_{\mathrm{r}}$ $=\Delta H_{\mathrm{r}}^{\circ}-T \Delta S_{\mathrm{r}}^{\circ}$, where $\Delta H_{\mathrm{r}}^{\circ}$ and $\Delta S_{\mathrm{r}}^{\circ}$ are the changes in the standard enthalpy and entropy for the reaction in question. At the same time, its relationship to electrochemical process can be described as $\Delta G_{\mathrm{r}}=-n F\left(V_{\text {th }}+\Delta V\right)$, where $n$ is the number of electrons transferred, $F$ is the Faraday's constant, and $\Delta V$ is the potential of standard hydrogen electrode relative to the ground of our equipment).

The values of $\Delta H_{\mathrm{r}}^{\circ}$ and $\Delta S_{\mathrm{r}}^{\circ}$ can be estimated by comparison to standard thermodynamic potentials of iron oxides. Oxidation of $\mathrm{FeO}$ to $\mathrm{Fe}_{2} \mathrm{O}_{3}$ leads to an enthalpy change of $-596 \mathrm{~kJ}$ and an entropy change of $-273 \mathrm{~J} / \mathrm{K}$ per $1 \mathrm{~mol}$ of reacted $\mathrm{O}_{2}$. The values for $\mathrm{FeO}$ oxidation to $\mathrm{Fe}_{3} \mathrm{O}_{4}$ are $-645 \mathrm{~kJ}$ and $-277 \mathrm{~J} / \mathrm{K}$ per $1 \mathrm{~mol}$ of $\mathrm{O}_{2}$, respectively (Hess's Law used for calculation). Note, that although the oxide products of the two reactions are different, values of $\Delta H_{\mathrm{r}}^{\circ}$ and $\Delta S_{\mathrm{r}}^{\circ}$ are very close. This allows us to estimate $\Delta H_{\mathrm{r}}^{\circ}$ and $\Delta S_{\mathrm{r}}^{\circ}$ as ca. $-60 \mathrm{~kJ} / \mathrm{mol}$ and $-30 \mathrm{~J} / \mathrm{mol} \cdot \mathrm{K}$, respectively, as for the oxidation of $1 \mathrm{~mol}$ of $10 \% \mathrm{CaBFO} x=$ 0.1 mols of $\mathrm{O}_{2}$ are required. Now

$$
V_{\text {th }}-\Delta V=-\frac{\Delta H_{\mathrm{r}}^{\circ}}{n F}+\frac{\Delta S_{\mathrm{r}}^{\circ}}{n F}
$$

Since each oxygen atom acquires 4 electrons and $x=0.1$ mols of oxygen molecules participate in reaction, $n=0.4$. Thus, calculation yields a slope of $V_{\text {th }}$ versus $T$ of about $7.7 \times 10^{-4} \mathrm{~V} /$ $\mathrm{K}$ and $V_{\text {th }}$ at $25{ }^{\circ} \mathrm{C}$ ca. $1.3 \mathrm{~V}$. The same slope calculated from Figure $9 \mathrm{~b}$ is 1.5 orders of magnitude higher $\left(1 \times 10^{-2} \mathrm{~V} / \mathrm{K}\right)$. Experimental $V_{\text {th }}$ at $25{ }^{\circ} \mathrm{C}$ is ca. $3.6 \mathrm{~V}$ (see Figure 9). This shows that the oxidation reaction on the gas-solid interface is unlikely to be the sole cause of resistive switching in Ca-BFO, and oxygen vacancy dynamics in the tip-surface junction plays the governing role on the kinetics of the process as was suggested before. ${ }^{52}$ Theoretical simulations based on the transport theory qualitatively confirm dependence of the FORC-IV loop opening on the oxygen vacancy diffusivity and value of the peak bias. It follows that the $I-V$ curves remain nonhysteretic below certain values of $V_{\mathrm{m}}$ and diffusivity, and loops progressively open up above them. The results are presented in the Supporting Information. 
IV. Summary and Outlook. In summary, we have demonstrated a new SPM-based method for probing local ionic and electronic transport and electrochemical phenomena in mixed ionic electronic conductors. The basic idea for the proposed ion flow imaging is a two-step first-order reversal curve measurement process based on current-voltage detection, or alternative detection schemes sensitive to the material properties coupled to oxygen vacancy concentration. The application of the FORC-IV method to Ca-BFO revealed uneven distribution of the conductive states and ionic activity in surface defects and nontrivial temperature dependence of the resistive switching dynamics. FORC-IV technique allowed separate probing of the temperature-activated transport of holes and oxygen vacancies, showing significant difference between the two.

The FORC-IV technique can be made more universal by modifications to the excitation waveform, including measurements with unipolar and bipolar waveforms and the use of lockin detection to record $\mathrm{d} I / \mathrm{d} V$ in addition to the current-voltage curves. It also allows performing dynamic measurements, such as sweep frequency-dependent that can be used to explore the dynamics of bias-induced transition and to acquire kinetic information. Implementation of active feedback (stop final current increase after loop opens to minimize damage and limit large-scale effects), and addition/deletion of opposite polarity cycles (after turn-on, to recover initial materials state assuming that we can have reasonably fast "erase" step) is also possible. Probing of the slow dynamics can be done by tuning the measurement frequency and/or bias sweep rate. For instance, a series of measurements can be taken with the same waveform bias peaks but different sweep rates, and the resulting FORCIVs can be plotted as a function of the sweep frequency to determine ionic kinetic information and/or ionic diffusion time at a specified location. Thus, this method can be extended to probe multiple relaxation times in mixed conductors. In conjunction with the electrochemical strain microscopy, FORC-IV can be used for differentiating ferroelectric and ionic behaviors. ${ }^{55}$

Finally, in the top-electrode setup, when the probing bias is applied between two planar macroscopic electrodes, establishing a lateral uniform electric field through the film, the SPM probe can act as a sensor of a local property changes related to the processes undergoing in the active material.

\section{ASSOCIATED CONTENT}

\section{S Supporting Information}

Additional details, Figures S1-S2, and Table S1. This material is available free of charge via the Internet at http://pubs.acs.org.

\section{AUTHOR INFORMATION}

\section{Corresponding Author}

*E-mail: (E.S.) strelcove@ornl.gov; (S.V.K.) sergei2@ornl.gov. Notes

The authors declare no competing financial interest.

\section{ACKNOWLEDGMENTS}

A part of this research was conducted at the Center for Nanophase Materials Sciences, which is sponsored at Oak Ridge National Laboratory by the Scientific User Facilities Division, Office of Basic Energy Sciences, U.S. Department of Energy. The work in National Chiao Tung University was supported by the National Science Council of Republic of
China (under contract No. NSC-101-2119-M-009-003-MY2), Ministry of Education (Grant MOE-ATU 101W961), and Center for Interdisciplinary Science at National Chiao Tung University.

\section{REFERENCES}

(1) Imada, M.; Fujimori, A.; Tokura, Y. Rev. Mod. Phys. 1998, 70, 1039.

(2) Dagotto, E. Science 2005, 309, 257.

(3) Pentcheva, R.; Pickett, A. E. J. Phys.: Condens. Matter 2010, 22.

(4) Burton, J. D.; Tsymbal, E. Y. Phys. Rev. Lett. 2011, 106.

(5) Tsymbal, E. Y.; Kohlstedt, H. Science 2006, 313, 181.

(6) Jeong, D. S.; Thomas, R.; Katiyar, R. S.; Scott, J. F.; Kohlstedt, H.; Petraru, A.; Hwang, C. S. Rep. Prog. Phys. 2012, 75, 076502.

(7) Fiebig, M. J. Phys. D: Appl. Phys. 2005, 38, R123.

(8) Spaldin, N. A.; Fiebig, M. Science 2005, 309, 391.

(9) Lottermoser, T.; Lonkai, T.; Amann, U.; Hohlwein, D.; Ihringer, J.; Fiebig, M. Nature 2004, 430, 541.

(10) Kimura, T.; Goto, T.; Shintani, H.; Ishizaka, K.; Arima, T.; Tokura, Y. Nature 2003, 426, 55.

(11) Biegalski, M. D.; Dorr, K.; Kim, D. H.; Christen, H. M. Appl. Phys. Lett. 2010, 96, 151905.

(12) Dekker, M. C.; Herklotz, A.; Schultz, L.; Reibold, M.; Vogel, K.; Biegalski, M. D.; Christen, H. M.; Dorr, K. Phys. Rev. B 2011, 84, 054463.

(13) Tselev, A.; Budai, J. D.; Strelcov, E.; Tischler, J. Z.; Kolmakov, A.; Kalinin, S. V. Nano Lett. 2011, 11, 3065.

(14) Kim, Y.; Kelly, J. S.; Morozovska, A.; Rahani, E. K.; Strelcov, E.; Eliseev, E.; Jesse, S.; Biegalski, M. D.; Balke, N.; Benedek, N.; Struckov, D.; Aarts, J.; Hwang, I.; Oh, S.; Choi, J. S.; Choi, T.; Park, B. H.; Shenoy, V.; Maksymovich, P.; Kalinin, S. V. Under review in Nano Letters.

(15) Lu, H.; Kim, D. J.; Bark, C. W.; Ryu, S.; Eom, C. B.; Tsymbal, E. Y.; Gruverman, A. Nano Lett. 2012, 12 (12), 6289-6292.

(16) Carter, S.; Selcuk, A.; Chater, R. J.; Kajda, J.; Kilner, J. A.; Steele, B. C. H. Solid State Ionics 1992, 53, 597.

(17) Petrov, A. N.; Cherepanov, V. A.; Zuev, A. Y. J. Solid State Electrochem. 2006, 10, 517.

(18) Tsipis, E. V.; Naumovich, E. N.; Patrakeev, M. V.; Yaremchenko, A. A.; Marozau, I. P.; Kovalevsky, A. V.; Waerenborgh, J. C.; Kharton, V. V. Solid State Ionics 2011, 192, 42.

(19) Pena, M. A.; Fierro, J. L. G. Chem. Rev. 2001, 101, 1981.

(20) tenElshof, J. E.; Lankhorst, M. H. R.; Bouwmeester, H. J. M. J. Electrochem. Soc. 1997, 144, 1060.

(21) Adler, S. B. Chem. Rev. 2004, 104, 4791.

(22) Kendall, S. C. S. a. K. High-temperature Solid Oxide Fuel Cells: Fundamentals, Design and Applications: Elsevier Science: New York, 2003.

(23) Kalinin, S. V.; Borisevich, A.; Fong, D. ACS Nano 2012, 6, 10423.

(24) Sawa, A. Mater. Today 2008, 11, 28.

(25) Strukov, D. B.; Snider, G. S.; Stewart, D. R.; Williams, R. S. Nature 2008, 453, 80.

(26) Valov, I.; Waser, R.; Jameson, J. R.; Kozicki, M. N. Nanotechnology 2011, 22.

(27) Szot, K.; Rogala, M.; Speier, W.; Klusek, Z.; Besmehn, A.; Waser, R. Nanotechnology 2011, 22.

(28) Waser, R.; Dittmann, R.; Staikov, G.; Szot, K. Adv. Mater. 2009, $21,2632$.

(29) Mamin, R. F.; Bdikin, I. K.; Kholkin, A. L. Appl. Phys. Lett. 2009, 94, 222901.

(30) Cheng, G. L.; Siles, P. F.; Bi, F.; Cen, C.; Bogorin, D. F.; Bark, C. W.; Folkman, C. M.; Park, J. W.; Eom, C. B.; Medeiros-Ribeiro, G.; Levy, J. Nat. Nanotechnol. 2011, 6, 343.

(31) Bristowe, N. C.; Littlewood, P. B.; Artacho, E. Phys. Rev. B 2011, $83,20$.

(32) Granquist, C. G. Sol. Energy Mater. Sol. Cells 2012, 99, 1. 
(33) Seidel, J.; Luo, W.; Suresha, S. J.; Nguyen, P. K.; Lee, A. S.; Kim, S. Y.; Yang, C. H.; Pennycook, S. J.; Pantelides, S. T.; Scott, J. F.; Ramesh, R. Nat. Commun. 2012, 3, 799.

(34) Scott, J. F. Solid State Ionics 1999, 125, 141.

(35) Morozovska, A. N.; Eliseev, E. A.; Svechnikov, G. S.; Kalinin, S. V. Phys. Rev. B 2011, 84, 045402.

(36) Morozovska, A. N.; Eliseev, E. A.; Tagantsev, A. K.; Bravina, S. L.; Chen, L. Q.; Kalinin, S. V. Phys. Rev. B 2011, 83, 195313.

(37) Kumar, A.; Ciucci, F.; Morozovska, A. N.; Kalinin, S. V.; Jesse, S. Nature Chem. 2011, 3, 707.

(38) Balke, N.; Jesse, S.; Morozovska, A. N.; Eliseev, E.; Chung, D. W.; Kim, Y.; Adamczyk, L.; Garcia, R. E.; Dudney, N.; Kalinin, S. V. Nat. Nanotechnol. 2010, 5, 749.

(39) Balke, N.; Jesse, S.; Kim, Y.; Adamczyk, L.; Tselev, A.; Ivanov, I.

N.; Dudney, N. J.; Kalinin, S. V. Nano Lett. 2010, 10, 3420.

(40) Kalinin, S.; Balke, N.; Jesse, S.; Tselev, A.; Kumar, A.; Arruda, T. M.; Guo, S. L.; Proksch, R. Mater. Today 2011, 14, 548.

(41) Jesse, S.; Balke, N.; Eliseev, E.; Tselev, A.; Dudney, N. J.; Morozovska, A. N.; Kalinin, S. V. ACS Nano 2011, 5, 9682.

(42) Bonnell, D. A.; Kalinin, S. V.; Kholkin, A. L.; Gruverman, A. Mrs Bulletin 2009, 34, 648.

(43) Gruverman, A.; Kholkin, A. Rep. Prog. Phys. 2006, 69, 2443.

(44) Kalinin, S. V.; Rar, A.; Jesse, S. IEEE Trans. Ultrason. Ferroelectr. Freq. Control 2006, 53, 2226.

(45) Balke, N.; Bdikin, I.; Kalinin, S. V.; Kholkin, A. L. J. Am. Ceram. Soc. 2009, 92, 1629.

(46) Kalinin, S. V.; Morozovska, A. N.; Chen, L. Q.; Rodriguez, B. J. Rep. Prog. Phys. 2010, 73, 056502.

(47) Bark, C. W.; Sharma, P.; Wang, Y.; Baek, S. H.; Lee, S.; Ryu, S.; Folkman, C. M.; Paudel, T. R.; Kumar, A.; Kalinin, S. V.; Sokolov, A.; Tsymbal, E. Y.; Rzchowski, M. S.; Gruverman, A.; Eom, C. B. Nano Lett. 2012, 12, 1765.

(48) Jang, H. W.; Kumar, A.; Denev, S.; Biegalski, M. D.; Maksymovych, P.; Bark, C. W.; Nelson, C. T.; Folkman, C. M.; Baek, S. H.; Balke, N.; Brooks, C. M.; Tenne, D. A.; Schlom, D. G.; Chen, L. Q.; Pan, X. Q.; Kalinin, S. V.; Gopalan, V.; Eom, C. B. Phys. Rev. Lett. 2010, 104, 197601.

(49) Kim, Y.; Kumar, A.; Tselev, A.; Kravchenko, I. I.; Han, H.; Vrejoiu, I.; Lee, W.; Hesse, D.; Alexe, M.; Kalinin, S. V.; Jesset, S. ACS Nano 2011, 5, 9104.

(50) Kalinin, V. S.; Balke, N.; Borisevich, Y. A.; Jesse, S.; Maksymovich, P.; Kim, Y.; Strelcov, E. Real Space Mapping of Oxygen Vacancy Diffusion and Electrochemical Transformations by Hysteretic Current Reversal Curve Measurements. Filed patent. 2012.

(51) Strukov, D. B.; Williams, R. S. Appl. Phys. A 2011, 102, 851.

(52) Yang, C. H.; Seidel, J.; Kim, S. Y.; Rossen, P. B.; Yu, P.; Gajek, M.; Chu, Y. H.; Martin, L. W.; Holcomb, M. B.; He, Q.; Maksymovych, P.; Balke, N.; Kalinin, S. V.; Baddorf, A. P.; Basu, S. R.; Scullin, M. L.; Ramesh, R. Nat. Mater. 2009, 8, 485.

(53) Wakiya, N.; Tajiri, N.; Kiguchi, T.; Mizutani, N.; Cross, J. S.; Shinozaki, K. Jpn. J. Appl. Phys., Part 2 2006, 45, L525.

(54) Orlov, A.; Kostishko, B.; Gonchar, L. Tech. Phys. Lett. 1998, 24, 81.

(55) Kim, Y.; Strelcov, E.; Hwang, I. R.; Choi, T.; Park, B. H.; Jesse, S.; Kalinin, S. V. Under review in Scientific Reports. 